\title{
Supporting Information: Low-Energy Phases of Bi Monolayer Predicted by Structure Search in Two Dimensions
}

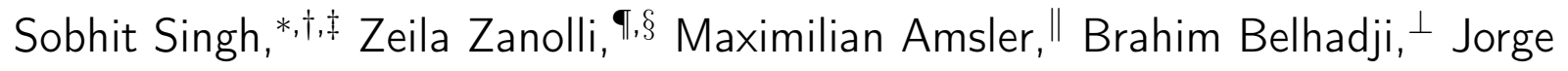
O. Sofo, $\#$ Matthieu J. Verstraete, ${ }^{\perp, \uparrow}$ and Aldo H. Romero*,†

$\dagger$ Department of Physics and Astronomy, West Virginia University, Morgantown, WV 26505, USA

$\ddagger$ Department of Physics and Astronomy, Rutgers University, Piscataway, NJ 08854, USA TCatalan Institute of Nanoscience and Nanotechnology (ICN2) and European Theoretical Spectroscopy Facility, CSIC and BIST, Campus UAB, Bellaterra, 08193 Barcelona, Spain §Institute for Theoretical Solid State Physics, RWTH Aachen University, D-52056 Aachen, Germany

||Laboratory of Atomic and Solid State Physics, Cornell University, Ithaca, New York 14853, USA

$\perp$ NanoMat/Q-Mat/CESAM and European Theoretical Spectroscopy Facility, Université de Liège (B5), B-4000 Liège, Belgium

\#Department of Physics and Materials Research Institute, The Pennsylvania State University, University Park, PA 16802, USA

E-mail: sobhit.singh@rutgers.edu; Aldo.Romero@mail.wvu.edu 
This Supporting Information (SI) contains details of the optimized crystal structures for all the six studied Bi monolayers; atomic orbital resolved electronic band structure; the spin-texture calculated at constant energy surfaces for the $\gamma$ phase; electronic band structure calculated without spin-orbit coupling (SOC) effects; topological characterization and edge states; strain-stabilized phonon spectrum for the $\gamma$-phase; a list of potential substrates for the $\alpha$-phase; and density of states (DOS). It also contains the electronic band structure of $b$-hexa, $p$-mono, and flat hexagonal Bi monolayer ( $f$-hexa) calculated considering $\mathrm{Bi} 5 d^{10}$ electrons in the core.

\section{Role of semicore $\mathrm{Bi}-5 d$ electrons on the electronic band structure}

Figure 1 shows the electronic band structure of $b$-hexa and $p$-mono phases calculated with SOC, considering $\mathrm{Bi} 5 d^{10}$ electrons in the core. The optimized lattice parameters using this pseudopotential are $4.38 \AA$ and $6.71 \AA$ for $b$-hexa and $p$-mono monolayers, respectively. The resulting Bi-Bi bond length is: $d=3.07 \AA$ for $b$-hexa phase, and $d_{\text {in-plane }}=$ $3.10 \AA, d_{\text {out-of-plane }}=3.10 \AA$ for $p$-mono monolayer structure. The inclusion of $5 d^{10}$ valence electrons is crucial to produce accurate results for $\mathrm{Bi}$ : it does not change the shape of the electronic bands near the Fermi level, but it does affect the overall bandgap very strongly. It also considerably changes the optimized lattice parameters of $\mathrm{Bi}$ (see the main text for more details).

To ensure that the observed $5 d$-electron potential does not suffer from additional pathologies (e.g. from missing, even deeper, semi-core states) we check the VASP calculations against all electron LAPW results, using the ELK code. We use standard input parameters and atomic configuration files, include spin orbit coupling, and employ the same geometry relaxed using the plane wave code. Figure 2 shows the electronic band structure within LAPW with SOC for the $f$-hexa structure. 

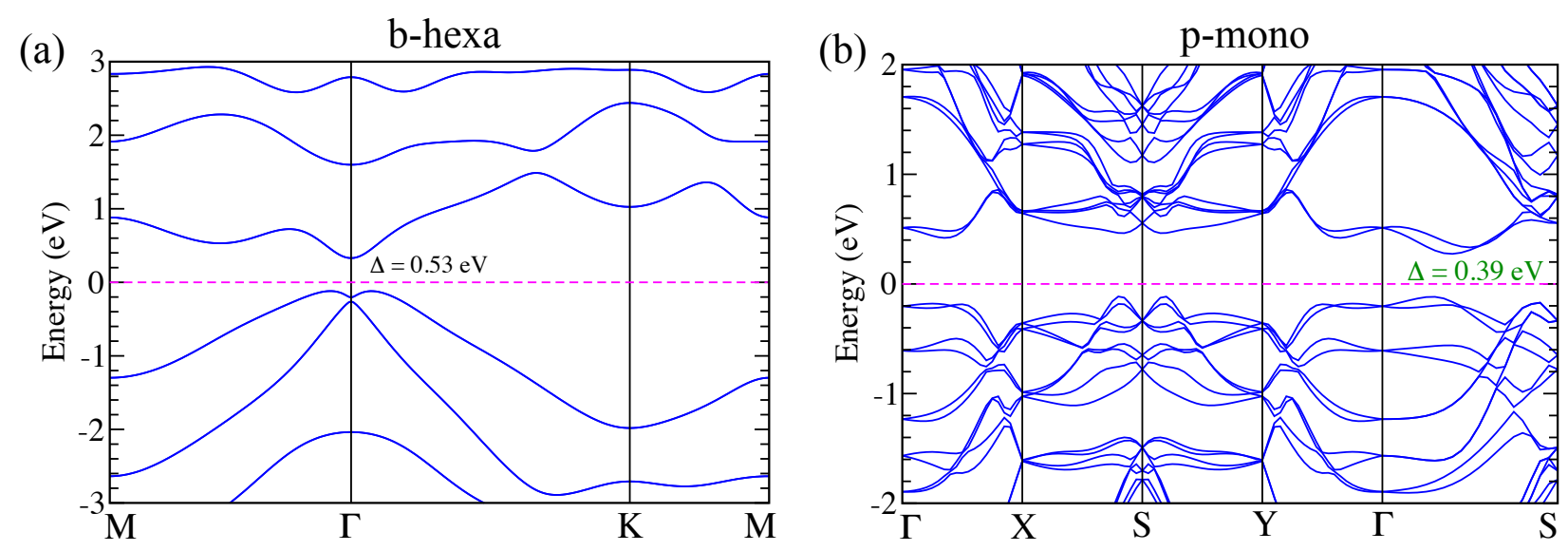

Figure 1: Electronic band structure $(\mathrm{PBE}+\mathrm{SOC})$ calculated considering $\mathrm{Bi} 5 d^{10}$ electrons in the core for (a) $b$-hexa, and (b) $p$-mono monolayers.

We also compare to the flat Bi monolayer structure used by Reis et al. [Science 2017, 357, 287âĂŞ290]. Note that no substrate included here, and lattice spacing is $5.35 \AA$. Upon flattening, the gap shifts to $\mathrm{K}$ and opens significantly, to a value coincidentally close to that at $\Gamma$ in the $b$-hexa phase, without $d$-electrons in the valence. We notice negligible changes in the electronic band structure of flat Bi monolayer with inclusion of $5 d$ electrons in valence, as shown in Figure 3. This confirms that the Bi $5 d$ electrons play a crucial role in determining the electronic band structure of Bi monolayer through the crystal field effects, which are negligible in the $f$-hexa phase. 


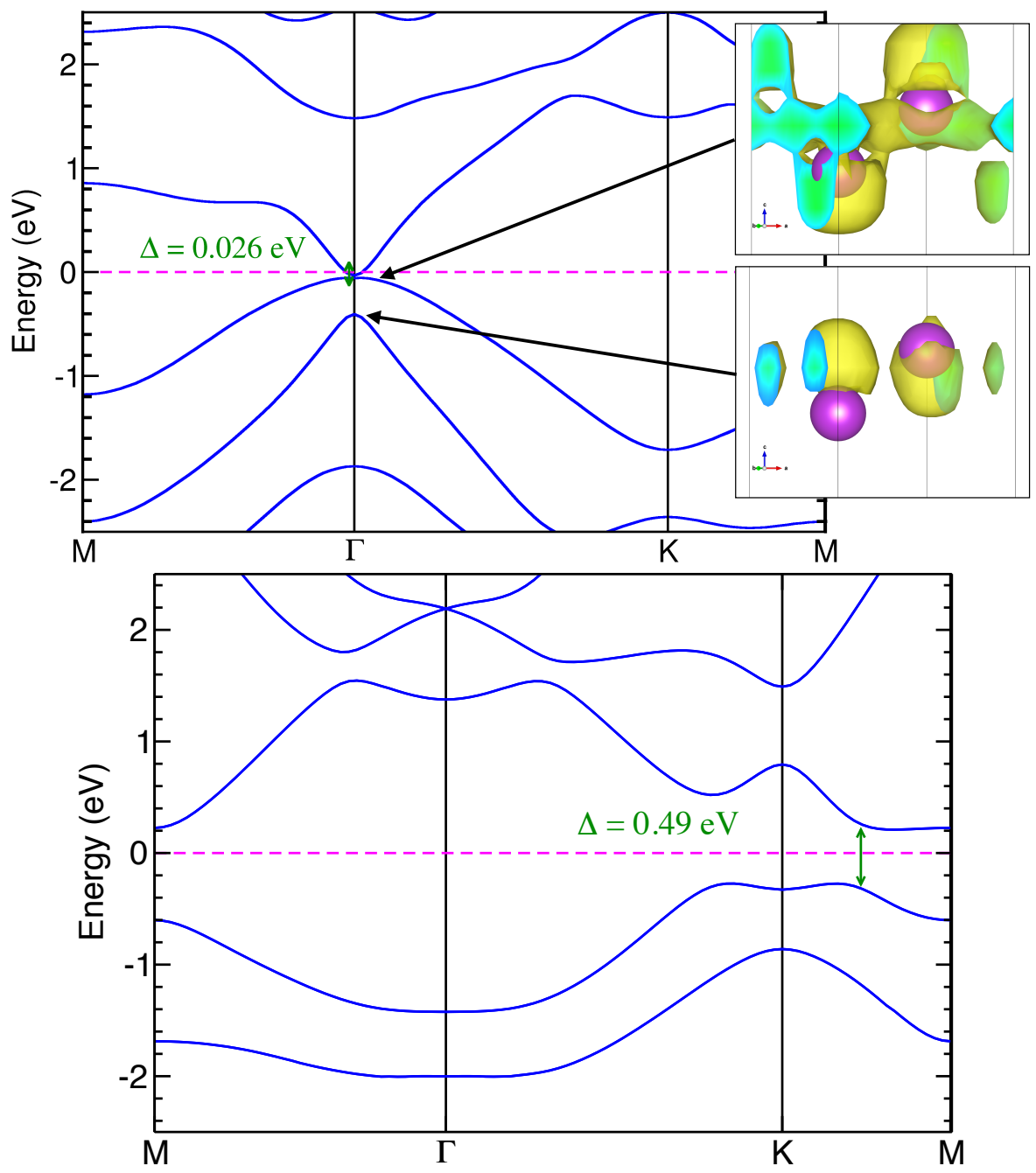

Figure 2: Electronic band structure (with SOC) calculated with the all-electron LAPW method and the ELK code. Top: $b$-hexa, with isodensities of the last two valence states at $\Gamma$. These show clear dangling bonds inwards and outwards from the buckled layer, which are sensitive to the presence of semi-core d electrons. Bottom: Flattened (graphene analog) $f$-hexa phase monolayer. 


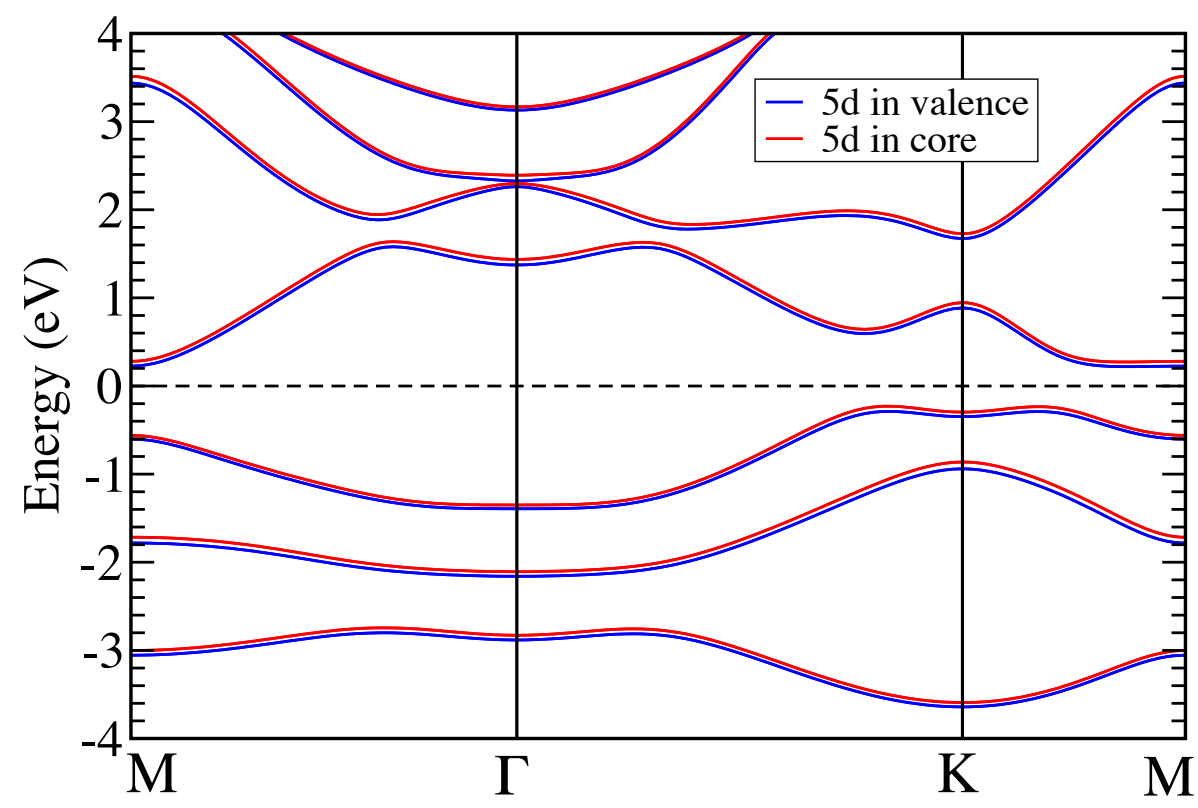

Figure 3: Role of Bi $5 d$ electrons on the electronic band structure of flat Bi monolayer $(f$ hexa) calculated with SOC.
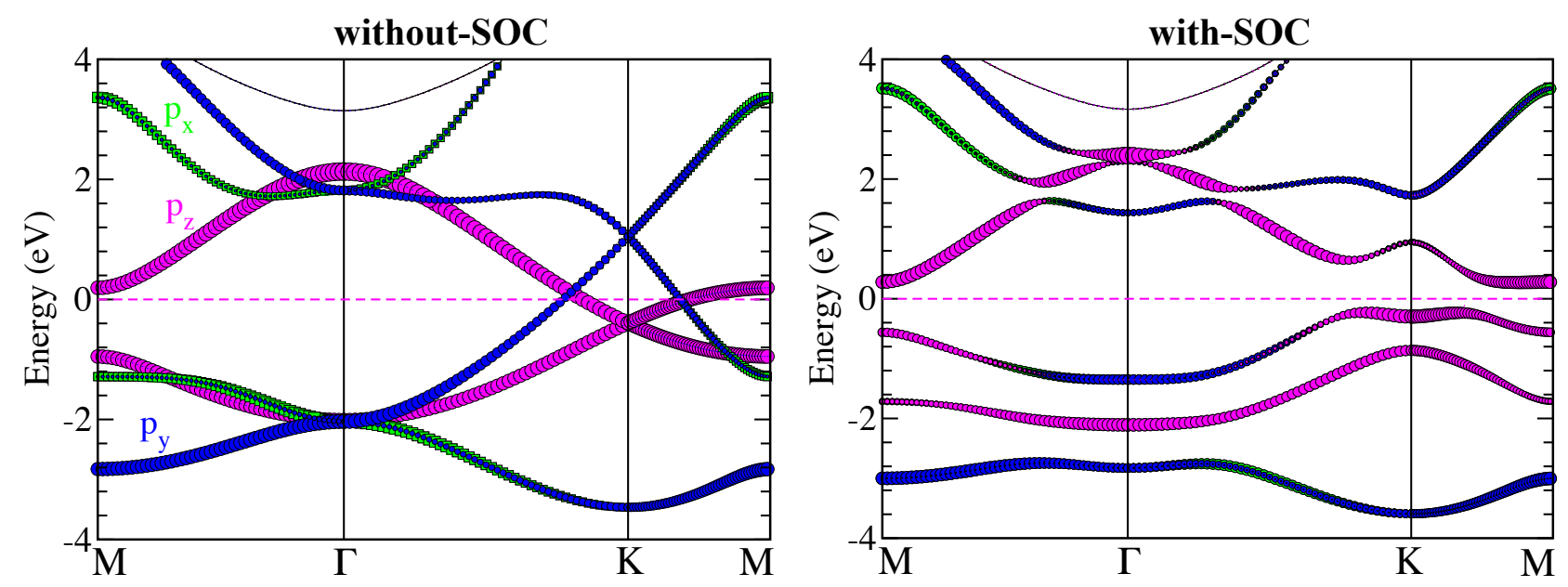

Figure 4: Orbital resolved electronic band structure of flat Bi monolayer ( $f$-hexa) calculated without (left) and with SOC (right) considering $5 d$ electrons in core. 


\section{Optimized lattice parameters and fractional coordinates of atoms}

Table 1: Puckered monoclinic structure

\begin{tabular}{llll}
\hline Lattice vectors $(\AA)$ & $\mathrm{x}$ & $\mathrm{y}$ & $\mathrm{z}$ \\
\hline $\mathbf{a}$ & 6.722 & -0.077 & 0.000 \\
$\mathbf{b}$ & -0.393 & 6.710 & 0.000 \\
$\mathbf{c}$ & 0.000 & 0.000 & 15.981 \\
\hline & & & \\
\hline Fractional coordinates & $\mathrm{x}$ & $\mathrm{y}$ & $\mathrm{z}$ \\
\hline $\mathrm{Bi}$ & 0.9679 & 0.4643 & 0.3728 \\
$\mathrm{Bi}$ & 0.5076 & 0.9239 & 0.5650 \\
$\mathrm{Bi}$ & 0.9677 & 0.9642 & 0.5920 \\
$\mathrm{Bi}$ & 0.4677 & 0.4642 & 0.5920 \\
$\mathrm{Bi}$ & 0.0076 & 0.4239 & 0.5650 \\
$\mathrm{Bi}$ & 0.5078 & 0.4240 & 0.3997 \\
$\mathrm{Bi}$ & 0.4679 & 0.9643 & 0.3728 \\
$\mathrm{Bi}$ & 0.0078 & 0.9240 & 0.3998 \\
\hline
\end{tabular}

Table 2: Buckled hexagonal structure

\begin{tabular}{llll}
\hline Lattice vectors $(\AA)$ & $\mathrm{x}$ & $\mathrm{y}$ & $\mathrm{z}$ \\
\hline $\mathbf{a}$ & 4.598 & 0.000 & 0.000 \\
$\mathbf{b}$ & -2.299 & 3.982 & 0.000 \\
$\mathbf{c}$ & 0.000 & 0.000 & 20.000 \\
\hline & & & $\mathrm{z}$ \\
\hline Fractional coordinates & $\mathrm{x}$ & $\mathrm{y}$ & 0.0591 \\
\hline $\mathrm{Bi}$ & 0.6667 & 0.3333 & 0.1408 \\
$\mathrm{Bi}$ & 0.0000 & 0.0000 & \\
\hline
\end{tabular}


Table 3: $\alpha$-phase

\begin{tabular}{llll}
\hline Lattice vectors $(\AA)$ & $\mathrm{x}$ & $\mathrm{y}$ & $\mathrm{z}$ \\
\hline $\mathbf{a}$ & 3.561 & 0.000 & 0.000 \\
$\mathbf{b}$ & 0.334 & 5.768 & 0.000 \\
$\mathbf{c}$ & 0.163 & -0.222 & 16.497 \\
\hline & & & \\
\hline Fractional coordinates & $\mathrm{x}$ & $\mathrm{y}$ & $\mathrm{z}$ \\
\hline $\mathrm{Bi}$ & 0.0776 & 0.5872 & 0.3966 \\
$\mathrm{Bi}$ & 0.5759 & 0.0873 & 0.4036 \\
$\mathrm{Bi}$ & 0.0810 & 0.5776 & 0.2050 \\
$\mathrm{Bi}$ & 0.5823 & 0.0781 & 0.2120 \\
\hline
\end{tabular}

Table 4: $\beta$-phase

\begin{tabular}{llll}
\hline Lattice vectors $(\AA)$ & $\mathrm{x}$ & $\mathrm{y}$ & $\mathrm{z}$ \\
\hline $\mathbf{a}$ & 7.955 & 0.000 & 0.000 \\
$\mathbf{b}$ & 3.232 & 7.258 & 0.000 \\
$\mathbf{c}$ & 0.000 & 0.000 & 16.000 \\
\hline & & & \\
\hline Fractional coordinates & $\mathrm{x}$ & $\mathrm{y}$ & $\mathrm{z}$ \\
\hline $\mathrm{Bi}$ & 0.7665 & 0.5962 & 0.2457 \\
$\mathrm{Bi}$ & 0.5352 & 0.8655 & 0.3793 \\
$\mathrm{Bi}$ & 0.8430 & 0.0196 & 0.3793 \\
$\mathrm{Bi}$ & 0.3430 & 0.5196 & 0.3793 \\
$\mathrm{Bi}$ & 0.0352 & 0.3655 & 0.3793 \\
$\mathrm{Bi}$ & 0.6121 & 0.2883 & 0.2457 \\
$\mathrm{Bi}$ & 0.2665 & 0.0962 & 0.2457 \\
$\mathrm{Bi}$ & 0.1121 & 0.7883 & 0.2457 \\
\hline
\end{tabular}

Table 5: $\gamma$-phase

\begin{tabular}{llll}
\hline Lattice vectors $(\AA)$ & $\mathrm{x}$ & $\mathrm{y}$ & $\mathrm{z}$ \\
\hline $\mathbf{a}$ & 7.613 & 0.000 & 0.000 \\
$\mathbf{b}$ & 2.086 & 7.321 & 0.000 \\
$\mathbf{c}$ & 0.000 & 0.000 & 16.000 \\
\hline & & & \\
\hline Fractional coordinates & $\mathrm{x}$ & $\mathrm{y}$ & $\mathrm{z}$ \\
\hline $\mathrm{Bi}$ & 0.8142 & 0.5676 & 0.2729 \\
$\mathrm{Bi}$ & 0.5631 & 0.8177 & 0.4030 \\
$\mathrm{Bi}$ & 0.8142 & 0.0676 & 0.3205 \\
$\mathrm{Bi}$ & 0.3142 & 0.5676 & 0.3199 \\
$\mathrm{Bi}$ & 0.0652 & 0.3167 & 0.4029 \\
$\mathrm{Bi}$ & 0.5651 & 0.3169 & 0.1897 \\
$\mathrm{Bi}$ & 0.3142 & 0.0676 & 0.2721 \\
$\mathrm{Bi}$ & 0.0632 & 0.8175 & 0.1897 \\
\hline
\end{tabular}


Table 6: Flat hexagonal ( $f$-hexa) phase

\begin{tabular}{llll}
\hline Lattice vectors $(\AA)$ & $\mathrm{x}$ & $\mathrm{y}$ & $\mathrm{z}$ \\
\hline $\mathbf{a}$ & 5.329 & 0.000 & 0.000 \\
$\mathbf{b}$ & -2.664 & 4.615 & 0.000 \\
$\mathbf{c}$ & 0.000 & 0.000 & 20.767 \\
\hline & & & \\
\hline Fractional coordinates & $\mathrm{x}$ & $\mathrm{y}$ & $\mathrm{z}$ \\
\hline $\mathrm{Bi}$ & 0.3333 & 0.6667 & 0.0000 \\
$\mathrm{Bi}$ & 0.6667 & 0.3333 & 0.0000 \\
\hline
\end{tabular}

\section{Electronic band structures calculated without SOC}

(a)

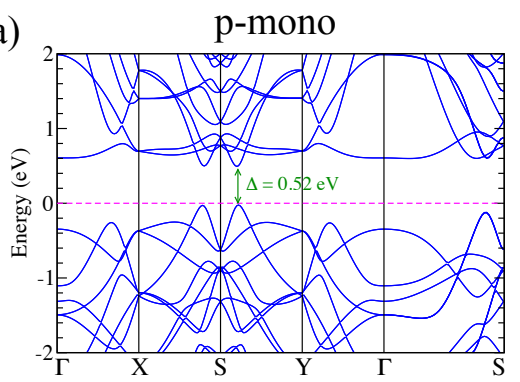

(d)

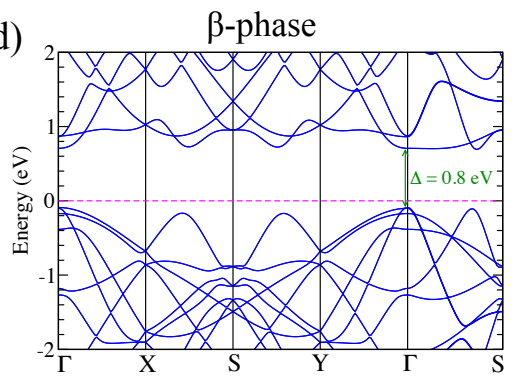

(b)

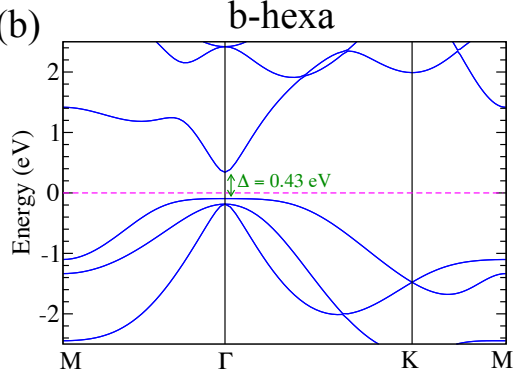

(e)

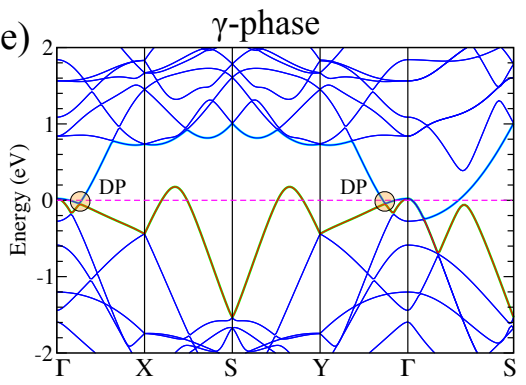

(c)

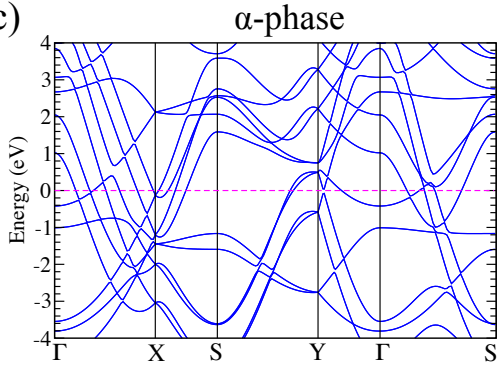

(f)

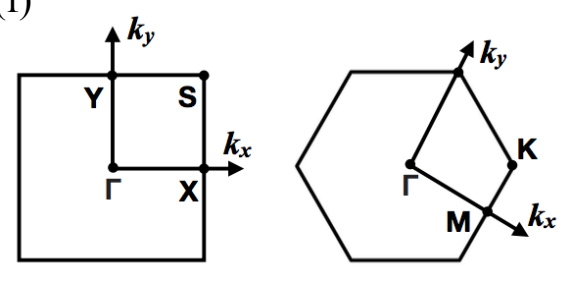

Figure 5: Electronic band structures calculated without considering SOC effects for (a) $p$ monoclinic, (b) b-hexagonal, (c) $\alpha^{-}$, (d) $\beta$-, and (e) $\gamma$-phases. (f) Brillouin zones marked with high-symmetry points. 


\section{Strain-stabilized phonon spectrum for $\gamma$-phase}

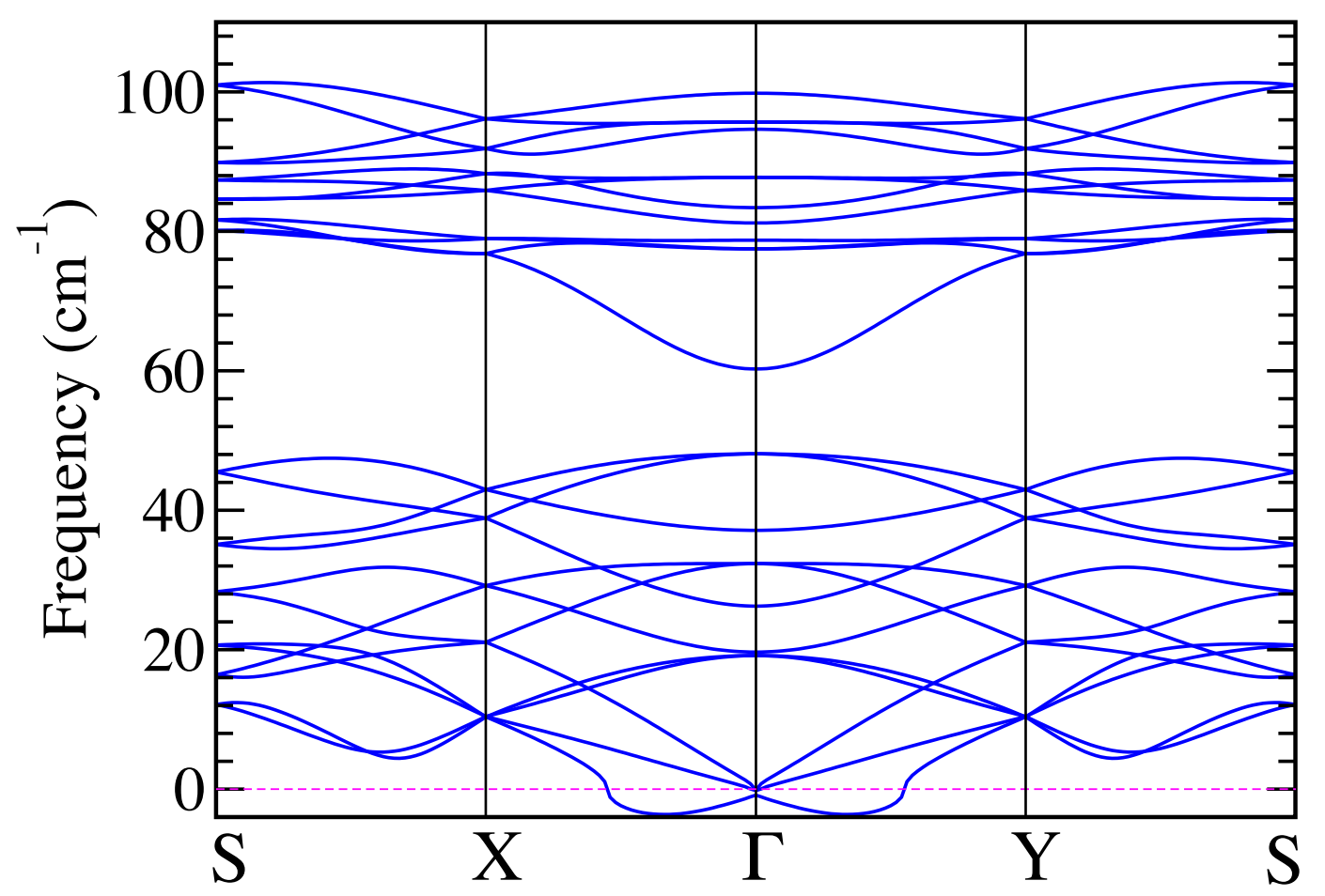

Figure 6: $8.0 \%$ tensile biaxial strain stabilized phonon spectrum of the $\gamma$-phase. The elastic instabilities near the $\Gamma$ points are due to poor numerical convergence at $k \rightarrow 0$ in $2 \mathrm{D}$ systems: the expected quadratic $2 \mathrm{D}$ mode is difficult to interpolate using Fourier methods, though all calculated frequencies are positive. The inner-coordinates were optimized after applying biaxial strain, and phonon spectrum was calculated using a supercell of size $2 \times 2 \times 1$. 


\section{Topological characterization}

\subsection{Inversion parity eigenvalues}

Table 7: The $Z_{2}$ topological invariant computed from the product of the inversion parity eigenvalues of all occupied bands at four TRIM points $\Gamma(0.0,0.0), M_{1}(0.5,0.0), M_{2}(0.0$, $0.5)$, and $M_{3}(0.5,0.5)$

\begin{tabular}{|c|c|c|}
\hline & Product of the parity eigenvalues & $Z_{2}$ \\
& $\Gamma M_{1} M_{2} M_{3}$ & \\
\hline$b$-hexa & -+++ & 1 \\
$f$-hexa & ++++ & 0 \\
$\alpha$-phase & -+++ & 1 \\
\hline
\end{tabular}

\subsection{Edge states}

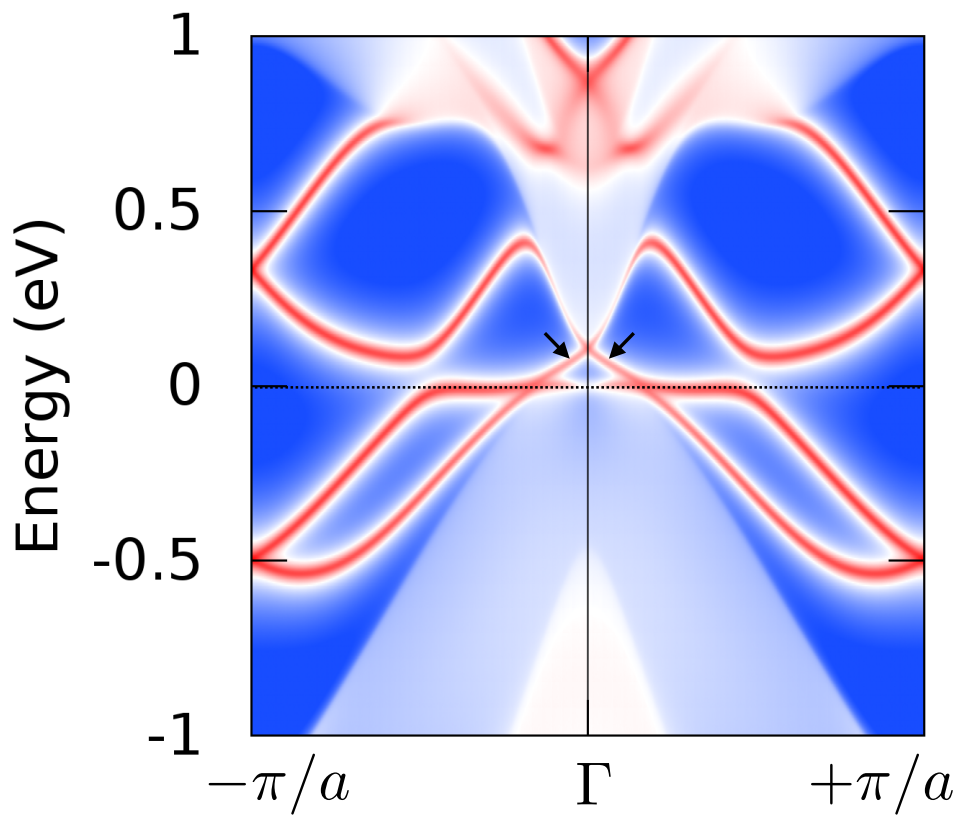

Figure 7: $b$-hexa phase: The edge state spectrum showing gapless dispersion of edge modes in the $b$-hexa phase. Arrows mark the topological nontrivial edge states. The flat edge states at the Fermi level are distinctive of the van Hove singularity. Red/Blue color represents the spectral density. Red color denotes the projection of edge states while white/blue color denotes the projection of monolayer states. 


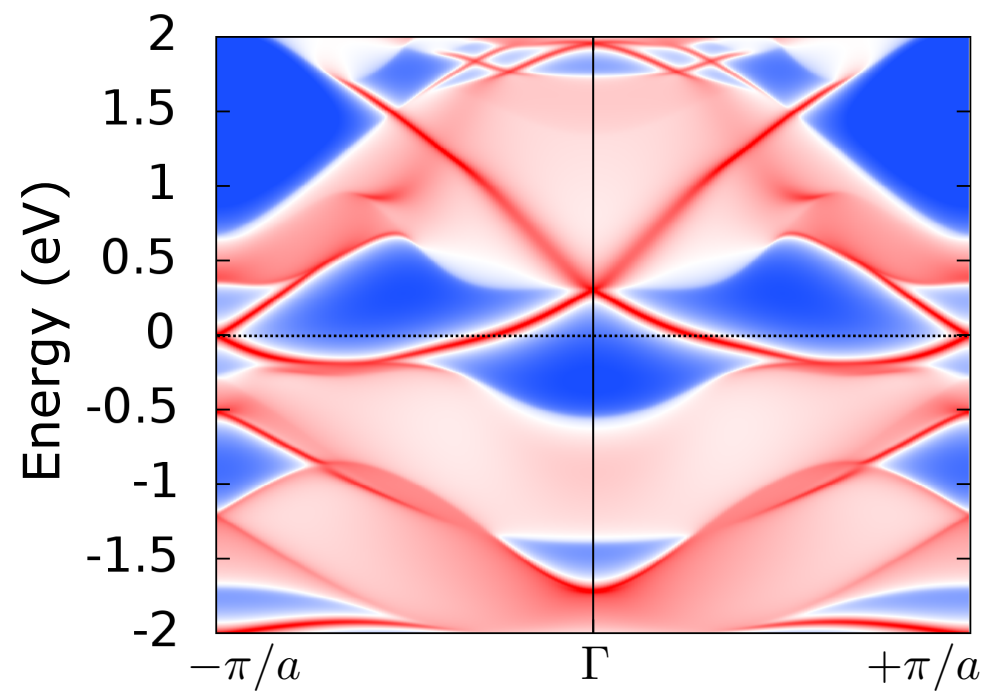

Figure 8: $f$-hexa phase: The edge state spectrum of $f$-hexa phase. Even number of edge states crossing the Fermi level along $\Gamma \rightarrow \pi / a$ kpath reveal the trivial topological nature of $f$-hexa phase, as predicted by calculated $Z_{2}$ topological invariant. Colors are as defined in Fig. 7. As noted by Reis et al. [Science 2017, 357, 287âĂŞ290], the topology becomes nontrivial once $\mathrm{SiC}$ substrate is included and planar mirror symmetry is broken. 

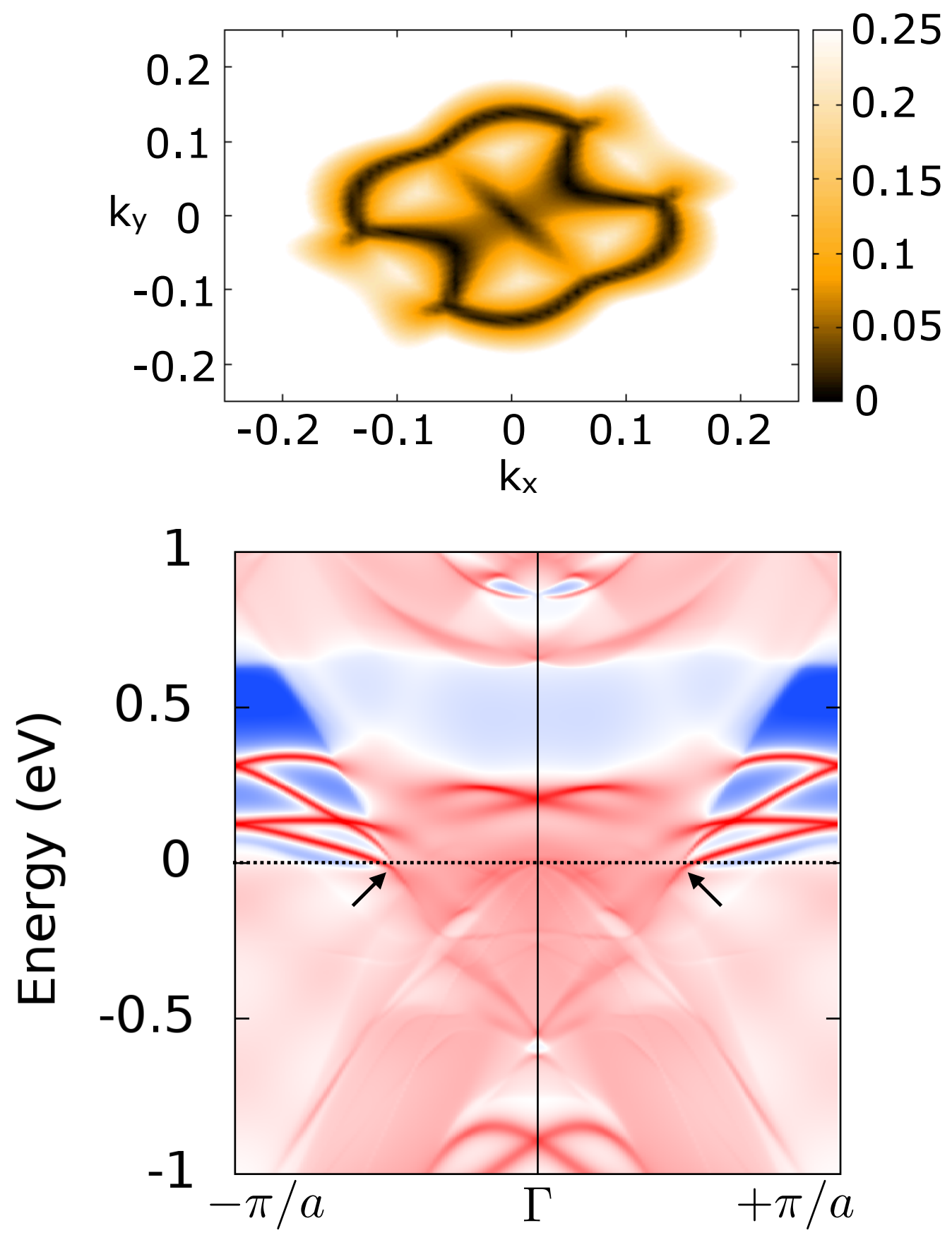

Figure 9: $\alpha$-phase: Top panel shows the energy bandgap plotted in color scale (eV units) in a $k_{x}-k_{y}$ plane centered at the $\Gamma$ point. A gapless Dirac nodal line can be noticed. Bottom panel depicts the edge state spectrum showing gapless nontrivial edge modes in the $\alpha$-phase. Arrows mark the topological nontrivial edge states originating from the Dirac point. 


\title{
6 Atomic orbital resolved band structure of $\gamma$ phase
}

\author{
$\gamma$-phase
}
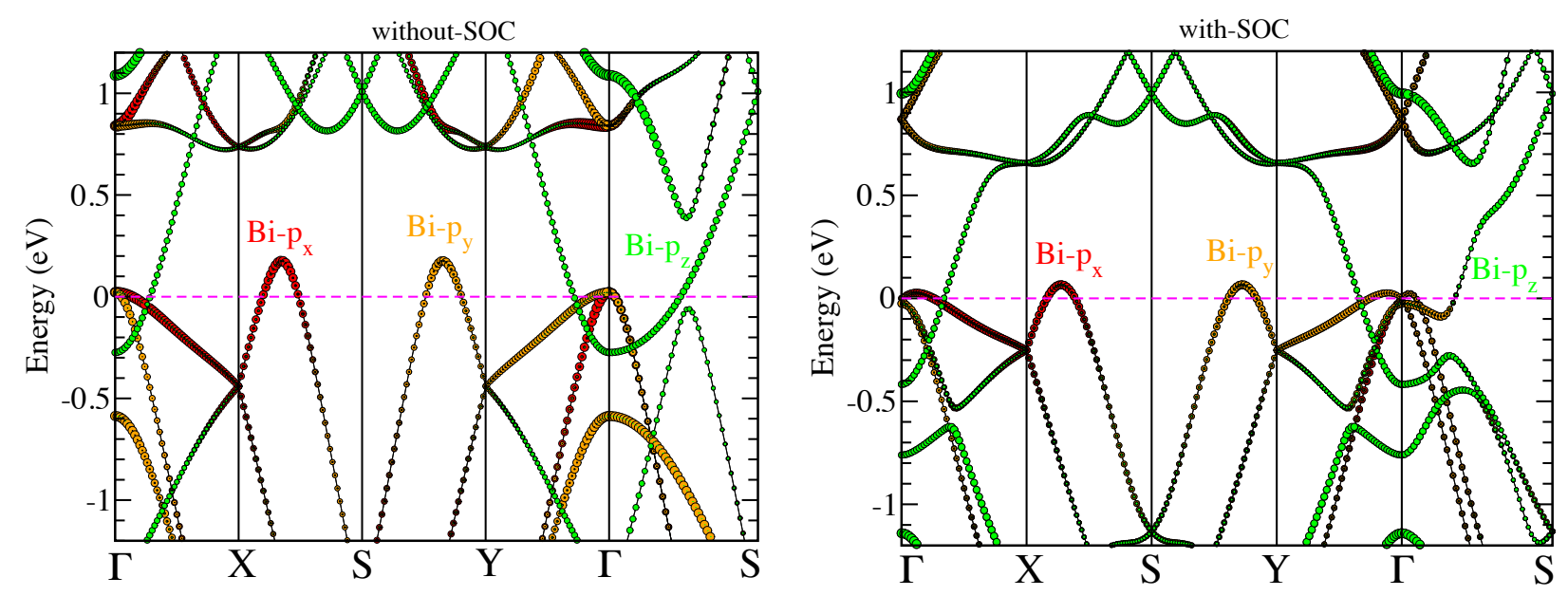

Figure 10: Atomic orbital resolved electronic band structure calculated without (left) and with (right) inclusion of SOC.

7 Spin-texture in $\gamma$ phase 


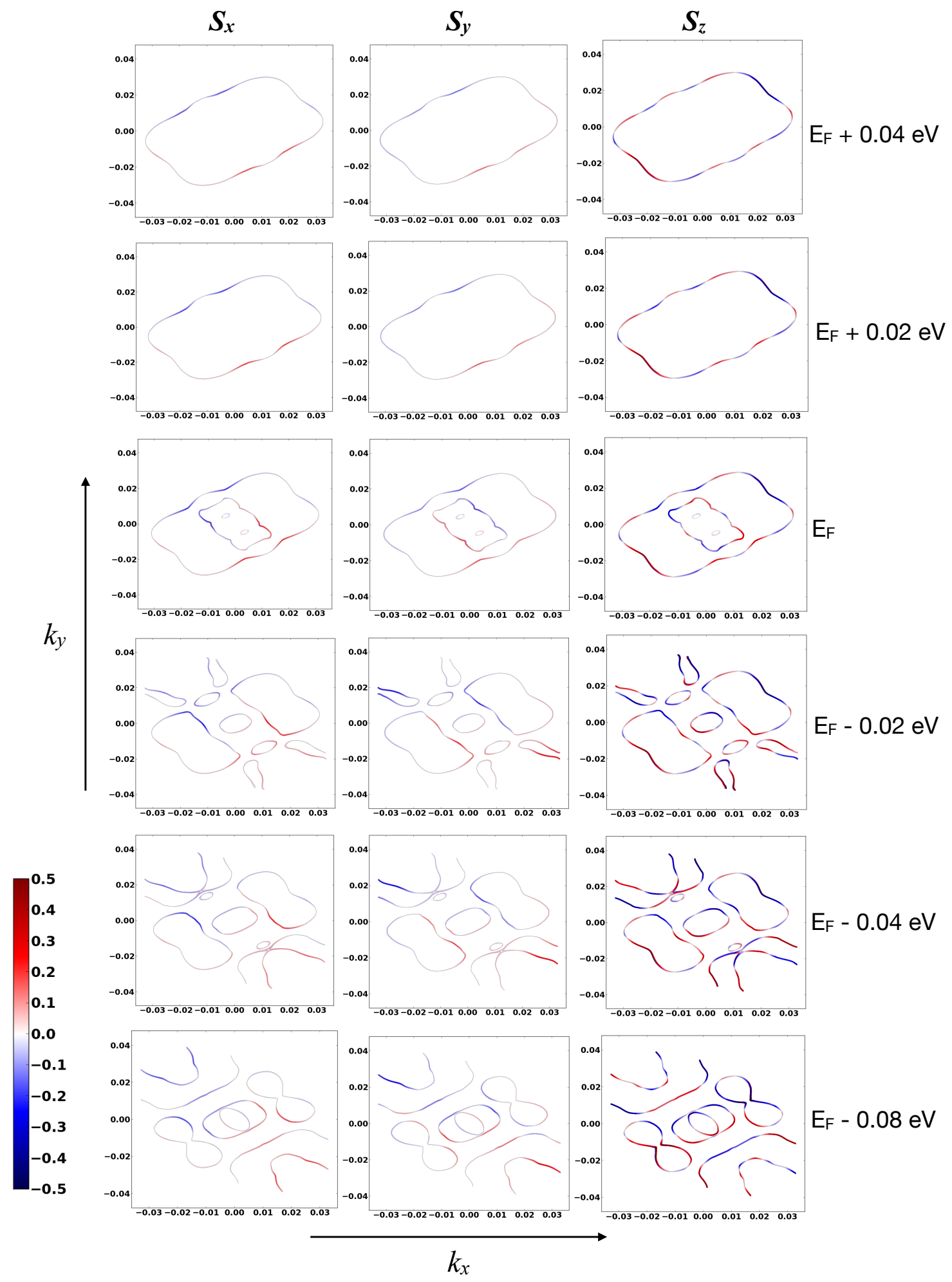

Figure 11: $\gamma$ phase : Spin-texture calculated at several different constant energy surfaces near the Fermi level in a $k_{x}-k_{y}$ plane centered at the $\Gamma$ point of Brillouin zone. 


\section{List of potential substrates for $\alpha$ phase}

Table 8: List of potential substrates for the $\alpha$ phase: This table shows a list of potential substrates that might be suitable for the synthesis of the $\alpha$ phase. For the substrate search, we employed the available algebraic algorithm provided by the MPInterfaces package, to search for geometrical matches with structures available in a material databases. We calculate the work-function of the matched substrates, and use it as a criterion to define a good substrate. The maximum area mismatch between the substrate candidates and $\mathrm{Bi}$ monolayer was set

to $40 \AA$, and cutoff for the maximum lattice mismatch was set to be $0.05 \AA$ with a maximum cell angle difference of $1^{\circ}$.

\begin{tabular}{cc}
\hline Compound & space group number \\
\hline $\mathrm{LiS}_{2} \mathrm{Ti}$ & 164 \\
$\mathrm{PtS}_{2}$ & 164 \\
$\mathrm{Mg}_{2} \mathrm{~Tb}_{4}$ & 164 \\
$\mathrm{Fe}_{6} \mathrm{Se}_{8}$ & 12 \\
$\mathrm{SnS}_{2}$ & 164 \\
$\mathrm{Mg}_{2} \mathrm{Y}_{6}$ & 44 \\
$\mathrm{As}_{2} \mathrm{Cr}_{2}$ & 186 \\
$\mathrm{MgB}_{2}$ & 191 \\
$\mathrm{PbO}$ & 187 \\
$\mathrm{Mn}_{4} \mathrm{Te}_{4}$ & 62 \\
$\mathrm{InTe}_{2}$ & 123 \\
$\mathrm{As}_{3} \mathrm{Co}_{3} \mathrm{Fe}_{3}$ & 189 \\
$\mathrm{PbS}$ & 166 \\
$\mathrm{Fe}_{2} \mathrm{Se}_{2}$ & 194 \\
$\mathrm{Tl}_{12} \mathrm{O}_{6}$ & 166 \\
$\mathrm{Ni}_{2} \mathrm{~Tb}_{2} \mathrm{C}_{4}$ & 38 \\
$\mathrm{Mn}_{6} \mathrm{P}_{3}$ & 150 \\
$\mathrm{MgCl}_{2}$ & 164 \\
$\mathrm{As}_{4} \mathrm{Rh}_{4}$ & 62 \\
$\mathrm{TiSe}_{2}$ & 164 \\
\hline
\end{tabular}

\section{Density of States}




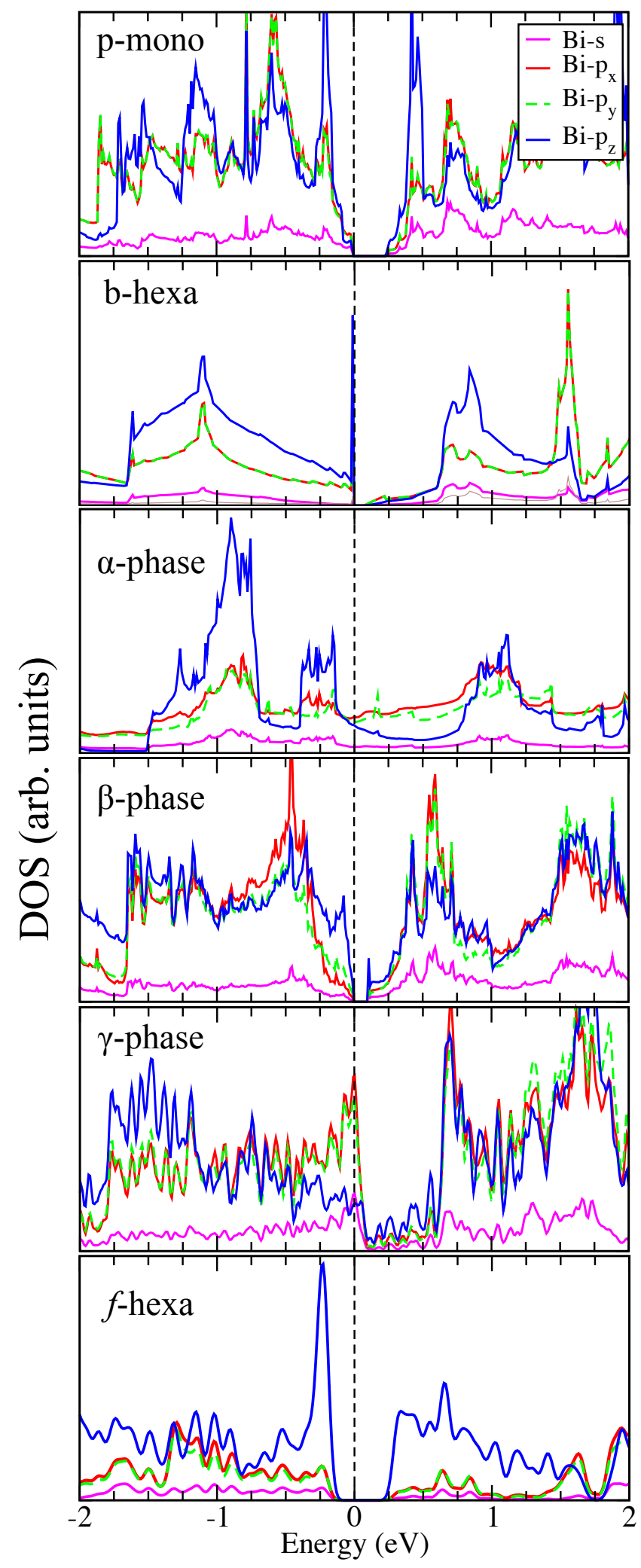

Figure 12: DOS calculated with SOC using a $k$-mesh of size $21 \times 21 \times 1$ for $p$-mono, $b$-hexa, $\alpha$-, $\beta$-, $\gamma$, and $f$-hexa phases of monolayer Bi (arranged in a top-to-bottom order, respectively). 